\title{
Asymmetric Core-collapse of a Rapidly-rotating Massive Star
}

\author{
Avishai Gilkis \\ Department of Physics, Technion - Israel Institute of Technology, Haifa 3200003, Israel \\ email: agilkis@technion.ac.il
}

\begin{abstract}
I find high turbulent shearing in a neutron-rich accretion disk surrounding a protoneutron star formed at the collapse of a rapidly-rotating $M_{\mathrm{ZAMS}}=54 M_{\odot}$ star. These might be features of superluminous supernovae powered by jets and/or magnetar spin-down.
\end{abstract}

Keywords. supernovae: general, stars: rotation

A $54 M_{\odot}$ star is evolved up to point of core-collapse with the MESA code (Paxton et al. 2011), with and without the Spruit dyanmo (Spruit 2002). The dynamo couples the core and envelope, so the loss of mass and angular momentum in winds affects the core and slows it down. At the onset of core-collapse the stellar models are mapped into the hydrodynamic solver FLASH (Fryxell et al. 2000), which includes core deleptonization and approximate neutrino transport (Couch \& O'Connor 2014). The three-dimensional flow is followed for $225 \mathrm{~ms}$ after core bounce. The rotation profile at stellar death significantly influences the flow dynamics and results of the collapse (Fig. 1). These results are also relevant for stellar evolution with magnetic fields where core spin-up occurs due to binary interaction, possibly resulting in a bipolar outflow.
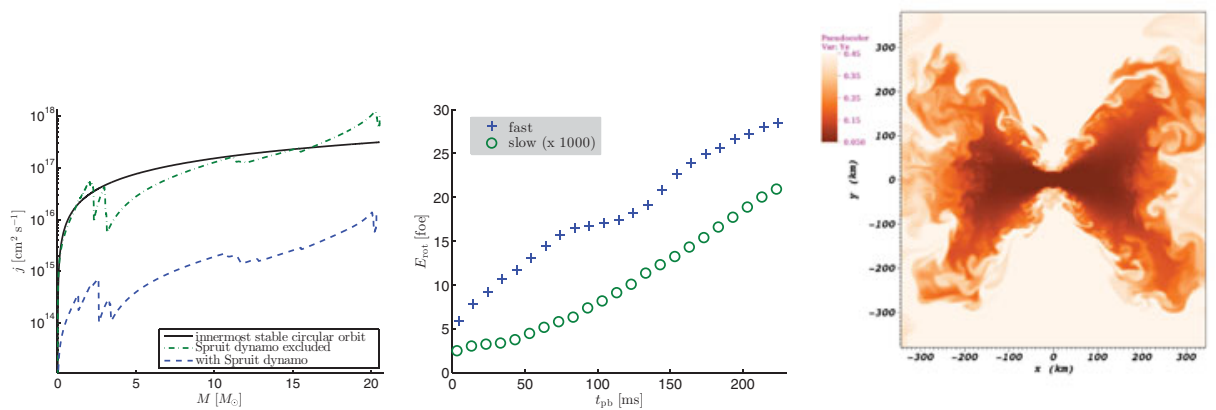

Figure 1. Left: Specific angular momentum as function of mass coordinate at the transition from MESA to FLASH. When the Spruit dynamo is excluded, the specific angular momentum is comparable to that of the innermost stable circular orbit around a Schwarzschild black hole. Center: Rotational kinetic energy of the proto-neutron star as function of time from core bounce. Right: Two-dimensional slice of the three-dimensional flow of the collapsing fast rotator $(y$ is the rotation axis) showing a disk of low electron fraction $\left(Y_{\mathrm{e}}\right)$ matter $200 \mathrm{~ms}$ after core bounce.

\section{References}

Couch, S. M. \& O'Connor, E. P. 2014, ApJ, 785, 123

Fryxell, B., et al. 2000, ApJS, 131, 273

Paxton, B., et al. 2011, ApJS, 192, 3

Spruit, H. C. 2002, A\&AA, 381, 923 